\title{
A Systematic Review on the Advancement in the Study of Fuzzy Variational Problems
}

\author{
Mansi Verma $\mathbb{D}^{1},{ }^{1}$ Chuei Yee Chen $\mathbb{D}^{1},{ }^{1}$ Adem Kılıçman $\mathbb{D}^{1,2}$ and Risman Mat Hasim $\mathbb{D}^{1}$ \\ ${ }^{1}$ Department of Mathematics and Statistics, Faculty of Science, Universiti Putra Malaysia, UPM Serdang, Selangor, Malaysia \\ ${ }^{2}$ Institute for Mathematical Research (INSPEM), Universiti Putra Malaysia, UPM Serdang, Selangor, Malaysia \\ Correspondence should be addressed to Chuei Yee Chen; cychen@upm.edu.my
}

Received 13 October 2021; Revised 27 December 2021; Accepted 29 December 2021; Published 7 February 2022

Academic Editor: Muhammad Gulzar

Copyright (C) 2022 Mansi Verma et al. This is an open access article distributed under the Creative Commons Attribution License, which permits unrestricted use, distribution, and reproduction in any medium, provided the original work is properly cited.

\begin{abstract}
The study of fuzzy variational problems has received significant attention over the past decade due to its successful applications in numerous fields, such as image segmentation and optimal control theory. The fuzzy Euler-Lagrange equations provide the necessary optimality conditions for solving fuzzy variational problems explicitly and have been studied under several differentiability conditions. In this paper, we provide a systematic review to recap the history of variational principle in the calculus of variations and compare it with the existing techniques in the fuzzy setting. We begin with the preliminary concepts and definitions of fuzzy theory and scrutinize the Euler-Lagrange's strategy via systematically searched studies concerning fuzzy variational problems to highlight the importance of improving the existing methods. Finally, we set up the main open problems regarding the limitations of the current approaches, shedding light on future directions.
\end{abstract}

\section{Introduction}

Over the past decade, there has been significant development in the study of fuzzy variational problems due to their practicality in implementation. One of its crucial roles is in the field of image processing, which in turn is used in medical imaging, geographic imaging, and forensic science [1-3]. The fuzzy variational problems are often solved numerically via Euler-Lagrange equations, Jacobi iterative techniques, or energy difference-based algorithms. One of such examples is the utilization of fuzzy Euler-Lagrange equations by Roul et al. [4] to provide solution for cost minimization while optimizing the production rate. In the study, the considered problem is the minimization of a fuzzy cost functional, where the integrand is in terms of production and stock functions which are fuzzy mappings. The model allows the decision-makers to decide how to optimize the production rate given different stock levels while keeping the cost at its optimal value. On the other hand, multicriteria decisionmaking (MCDM) has been well studied under fuzzy settheoretic models [5-10] to analyze and rank alternatives from the finest to the poorest criteria concerning decision- maker preferences [11]. Since most practical optimization problems can be modelled as fuzzy energy functionals, it is therefore advantageous to study fuzzy variational problems for decision-making. However, the existing studies in fuzzy variational problems are mostly aimed at obtaining the explicit solution by deriving the Euler-Lagrange equations in the fuzzy setting [12-14], which are essentially based on the intuition that every extremal problem will have a solution. This is in contrast to the standard variational principle in the calculus of variations that focuses on the existence of minimizers or more generally the existence of solutions.

Known as the classical method for solving variational problems in the calculus of variations, the history of the Euler-Lagrange approach can be traced back to one of the ancient problems in mathematics, namely, the isoperimetric problem or Dido's problem, that is to determine the curve of a given length which encloses a maximal area. Though a circle appeared to be an obvious solution to the isoperimetric problem, there was no rigorous mathematical proof until 1744 when Euler [15] revisited the isoperimetric problem. The problem was eventually solved by means of the standard methods in calculus. However, Euler noted the complicated 
process of his geometrical approach, which may require a more straightforward strategy in acquiring the variational conditions. In 1755, Euler received a letter from a nineteen-year-old Lagrange who had found a more general approach to the problem, which is purely analytical. Dropping the geometrical approach, Euler and Lagrange developed a systematic way to solve such variational problems which includes Dido's problem, Galileo's brachistochrone problem that was formulated in 1638, Fermat's optical problem in 1662, and Newton's problem on floating bodies in 1685. This leads to what we know today as the EulerLagrange equation and hence the birth of the calculus of variations.

In view of modern calculus of variations, the EulerLagrange equation is usually regarded as the classical method, and one of its disadvantages is that it was established based on the intuition that every extremal problem will have a solution. In particular, while being a solution to the Euler-Lagrange equation is necessary for the minimization problem, it is far from being a sufficient condition. This approach seems to be inadequate since there is no guarantee that a minimizer will exist. Moreover, the solutions are assumed to be regular (usually $C^{1}$ or $C^{2}$ ). These limitations led to another class of methods, namely the direct method in the calculus of variations, which is a powerful abstract method for proving the existence of minimizers. This approach grew out of many people's work; in particular, its early development is often attributed to the works of Riemann, Hilbert, Weierstrass, and Tonelli. The essence of the direct method is to show the existence of a minimizer of a given integral functional and subsequently to prove its regularity. Interested readers on the history of calculus of variations may refer to the books of Giaquinta and Hildebrant [16], Goldstine [17], and Monna [18].

Instead of finding the minimizers of variational problems directly, the direct method focuses on finding these minimizers implicitly by considering minimizing sequences and the concept of lower semicontinuity of the given integral functional. In 1920, Tonelli [19] proved that the functional is lower semicontinuous if and only if its integrand is twice continuously differentiable and convex in the last variable for the one-dimensional case. The work of Tonelli was further extended to the $n$-dimensional case by McShane [20]. However, though the regularity condition of the integrand assumed by McShane [20] and Tonelli [19] was sufficient, it was too strong to be true. Therefore, Serrin [21] tried to relax the requirement of differentiability under the assumptions of continuity and nonnegativity. Later, instead of differentiability and continuity, Marcellini and Sbordone [22] assumed the integrand to be a Carathéodory function (measurable in the first variable and jointly continuous in other variables) and gave the necessary and sufficient conditions for lower semicontinuity of the functional. This led to the general existence theorem with minimal regularity conditions. The variational principle in the calculus of variations has since been applied in studies that vary from the optimal design for thin films [23] to the construction of an ideal column [24] and from quantum field theory [25] to softer spacecraft landings [26]. Apart from that, the development in the calculus of variations has contributed to the advance- ment of multiple fields of mathematics, including topology, functional analysis, and partial differential equations $[27,28]$.

One of the fundamental drawbacks of crisp functionals in the calculus of variations is the assumption that all the variables defining a particular energy functional would be accessible accurately. This is a significant restriction since, in most practical cases, not all factors are quantifiable. Recent research studies in image segmentation varying from the detection of the objects in an image to contouring vessel structures highlight similar drawbacks of the conventional crisp functional models, where they are highly dependent on the initial images and are unable to handle objects with ill-defined boundaries $[29,30]$. Since not all results can be obtained in the crisp sense of Aristotelian logic due to the complexity of problems, the development of fuzzy functionals seems to be promising to handle such problems. In response to this, our study is aimed at discussing fuzzy functionals that consider both fuzzy logic and calculus of variations.

Many practical problems such as the propagation of wave, heat, fluid flow, and optimization theory [31] are formulated using partial differential equations that can be solved by various means of numerical and analytical methods. One of such methods is the variational method using the Lagrangian density function to solve the corresponding partial differential equations. Though there have been studies focusing on solving the fuzzy partial differential equations to model uncertainties and to study the existence of their solutions (see, for instance, [32-36]), there has been little attention on the variational principle for solving fuzzy variational problems. In recent years, some studies have been dedicated to establishing the necessary optimality conditions of fuzzy variational problems for finding the extremals $[12,13,37]$. However, the question remains to be considered is the existence of those extremals without resorting to the Euler-Lagrange equations.

Moreover, real-world problems like image segmentation and optimal control theory generally focus on the minimization of energy integrals that implicity correspond to fuzzy variational problems $[4,29,38]$. They are mainly addressed by iteration techniques under the assumption that a minimizer exists. Despite its importance, there is a lack of comprehensive literature that analyzes the study of the existence of minimizers and the sufficient conditions in the fuzzy setting. Thus, it is crucial to establish fuzzy variational principle such that the uncertainties in real-world problems can be addressed more efficiently. Table 1 summarizes the literature and research gaps that motivate this study as a standardized and systematic review to highlight the importance of the study of the existence of minimizer for fuzzy variational problems.

The outline of this paper goes as follows. In Section 2, we present the preliminary concepts and definitions of fuzzy theory that will be used throughout this paper. In Section 3, we discuss the Euler-Lagrange approach, followed by scrutinizing its methodology and presenting systematically searched studies concerning fuzzy variational problems to reveal the necessity for the improvement of such techniques. Finally, in Section 4, we set up 
TABLE 1: Review of the related fields and their research gaps.

\begin{tabular}{|c|c|c|}
\hline Related fields & Literature & Research gaps \\
\hline $\begin{array}{l}\text { Calculus of } \\
\text { variations }\end{array}$ & $\begin{array}{l}\text { The variational principle in the calculus of variations } \\
\text { has been applied in many real-world problems whenever } \\
\text { they can be mathematically formulated as a minimization } \\
\text { (or maximization) problem. Some of its applications } \\
\text { include softer spacecraft landings [26], optimal design } \\
\text { for thin films [23], and construction of an ideal column } \\
\text { [24], of which they are modelled as energy functionals } \\
\text { and solved using the variational principle. }\end{array}$ & $\begin{array}{l}\text { In reality, not all variables are deterministic. Recent } \\
\text { research studies have shown that the conventional } \\
\text { crisp functional models are insufficient in solving } \\
\text { image segmentation problems such as the detection of } \\
\text { objects in an image and vessel structures contouring, } \\
\text { since the accuracy of the models rely on the position } \\
\text { of the initial images }[29,30] \text {. It turns out that the } \\
\text { fuzzy models, which involve solving fuzzy energy } \\
\text { functionals, are able to extract the boundaries } \\
\text { accurately under flexible initialization. Despite } \\
\text { its applicability, the study of fuzzy variational } \\
\text { problems can be considered at its early stage. }\end{array}$ \\
\hline
\end{tabular}

Fuzzy variational problems have been solved via the Euler-Lagrange equations and numerically by Jacobi iterative techniques or energy difference-based algorithms. The necessary

Fuzzy variational principle optimality conditions given by the Euler-Lagrang equations were first derived by Farhadinia [13] using the Buckley-Fuering derivative approach [33] under twice differentiability assumptions. For similar results under different differentiability notions, one may refer to $[12,14,42]$.
Solving fuzzy variational problems via the fuzzy Euler-Lagrange equations relies on the smoothness of the integrand, which might not be available. Moreover, it is established based on the intuition that every extremal problem will have a regular solution. Such an assumption seems inadequate because there is no guarantee that a solution will exist. Therefore, it is more fruitful to address the existence of solutions in the fuzzy setting first.
Fuzzy decision-making: it aids researchers and practitioners in analyzing and ranking alternatives concerning decision-maker preferences [11, 43-45].

It has also been expanded and studied for many generalized classes of fuzzy sets under multiple parameters to address uncertain information more efficiently [5-10].

Applications

Image segmentation and optimal control theory: these problems are generally focused on the minimization of energy functionals that corresponds to a fuzzy variational problem $[4,29,38]$. They are usually solved numerically by Jacobi iterative techniques or energy difference-based algorithms, under the assumption that a minimizer exists.
Existing studies focus primarily on set-theoretic modeling. However, most of the optimization problems in practical life usually appear as fuzzy energy functionals that explain the need to study fuzzy variational problems.

Despite its importance, there has been a lack of studies to address the existence of solutions in a nonconstructive way. It is crucial to establish fuzzy variational principle such that the uncertainties in real-world problems can be handled more efficiently. the open problems with respect to the limitations of the current methodology to give an overview of possible directions for future research.

\section{Standard Definitions in Fuzzy Theory}

In this section, we present some standard definitions that follow from Dubo and Prade [39], Klir and Yuan [40], and Zimmermann [41]. These definitions would be used throughout the paper.

Definition 1 (crisp set). A set $A$ is called as a crisp set if there is no ambiguity regarding the inclusion or exclusion of its elements.

Example 1. Let $A$ be the set of all natural numbers. Any real number will be either an element of the set $A$ or not.

Definition 2 (fuzzy set and membership function). Let $X$ be the universe of discourse and $x$ represents its elements.
Then, a fuzzy set in $X$, denoted by $\tilde{A}$, is defined using a set of ordered pairs as follows:

$$
\tilde{A}=\left\{\left\{x, \mu_{\tilde{A}}(x)\right\}: x \in X\right\}
$$

where $\mu_{\tilde{A}}: X \longrightarrow[0,1]$ is called the membership function that gives a degree of membership to every element of $X$ ranging from 0 to 1 .

A fuzzy set is a mathematical way of representing ambiguous or subjective data obtained through human language. In a fuzzy set, an element can be partially contained in the set whenever its inclusion is ambiguous, and we represent it by the degree of membership that is between 0 and 1 . For instance, consider a collection of tall students of grade 12 from a school. Now, the term "tall" seems subjective. A person with a height of 5.4 feet might be tall to someone who is 5 feet, but a 6.3 feet tall person may not consider 5.4 feet to be tall. The word "tall" is ambiguous and 


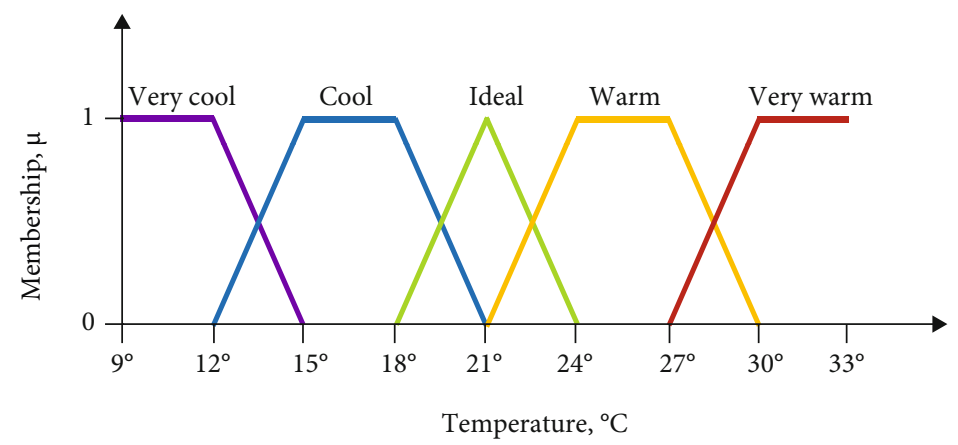

FIGURE 1: Fuzzy sets with different membership functions corresponding to the range of temperature.

subjective, and such a collection cannot be defined mathematically using the crisp set. Instead, it can be defined via fuzzy sets using linguistic language.

Similarly, let us consider the case of an automatic airconditioning system along with a temperature sensor working over a fuzzy algorithm to monitor the speed of a motor that maintains an ideal temperature [46]. A fuzzy inference system generally consists of fuzzification for giving fuzzy inputs, knowledge-based if-then rule evaluation followed by fuzzy outputs, and its defuzzification to obtain a single output of the aggregated fuzzy set. Fuzzification refers to the process of storing linguistic variables as fuzzy sets using membership functions. Figure 1 illustrates the linguistic representation of the air temperature using five fuzzy sets for temperature, namely, Very cool, Cool, Ideal, Warm, and Very warm.

With this fuzzy system, the transitions of temperature from one state to another would be easier as with the temperature change. Subsequently, that can be expressed via a corresponding change in the values of the membership functions. The microcontroller then makes decisions using ifthen rules based on human expertise to speed up or slow down the motor and adjust the temperature. In case, if it is warm, which is between 24 and 27 degrees, the speed of the motor is very low, and the temp is very high. In response to this, fuzzy inference system will lower the temperature and increase the speed of the motor. Such rule evaluations go inside the microcontroller of the system that results in a fuzzy output. The defuzzification fuzzy output is also done by the microcontroller to give a crisp solution. In this way, a fuzzy system based on knowledge-based if-then rule mimics human behavior that makes it more practical and applicable [47]. Some of the common membership functions used above can be stated mathematically as follows:

(1) Triangular Membership Function. The triangular membership function $\mathrm{MF}_{T}$ is given by

$$
\mathrm{MF}_{T}(x ; a, b, c)= \begin{cases}0, & \text { if } x \leq a, \\ \frac{x-a}{b-a}, & \text { if } a \leq x \leq b, \\ \frac{c-x}{c-b}, & \text { if } b \leq x \leq c, \\ 0, & \text { if } x \geq c,\end{cases}
$$

where $a, b$, and $c$ are the points with membership values 0,1 , and 0 , respectively. The graphical illustration for the fuzzy sets defined using $\mathrm{MF}_{T}$ is given in Figure 2.

(2) Trapezoidal Membership Function. The trapezoidal membership function $\mathrm{MF}_{T p}$ is given by

$$
\mathrm{MF}_{T p}(x ; a, b, c, d)= \begin{cases}0, & \text { if } x \leq a, \\ \frac{x-a}{b-a}, & \text { if } a \leq x \leq b, \\ 1, & \text { if } b \leq x \leq c, \\ \frac{d-x}{d-c}, & \text { if } c \leq x \leq d, \\ 0, & \text { if } x \geq d,\end{cases}
$$

where $a, b, c$, and $d$ are the points with membership values $0,1,1$, and 0 , respectively. The graphical illustration for the fuzzy sets defined using $\mathrm{MF}_{T p}$ is given in Figure 3.

(3) Gaussian Membership Function. The Gaussian membership function $\mathrm{MF}_{\mathrm{Tg}}$ is given by

$$
\operatorname{MF}_{T g}(x, c, \sigma)=\exp ^{-(1 / 2)((x-c) / \sigma)^{2}}
$$

where $c$ denotes the membership function center and $\sigma$ denotes its width. The graphical illustration for the fuzzy sets defined using $\mathrm{MF}_{T g}$ is given in Figure 4.

(4) Generalized Bell Membership Function. The generalized bell membership function $\mathrm{MF}_{T g *}$ is given by

$$
\mathrm{MF}_{T g *}=\frac{1}{1+|(x-c) / a|^{2 b}},
$$

where variable $b$ is usually positive. In the event when $b$ is negative, the shape of this $\mathrm{MF}_{T g *}$ turns to an upside-down. Moreover, $\mathrm{MF}_{T g *}$ is a straightforward generalization of the Cauchy distribution. Here, $a \neq 0$ controls the width of the bell shaped curve, $b$ is a positive integer responsible for the arch of the curve, and $c$ determines the center of the curve. For instance, consider a fuzzy set 


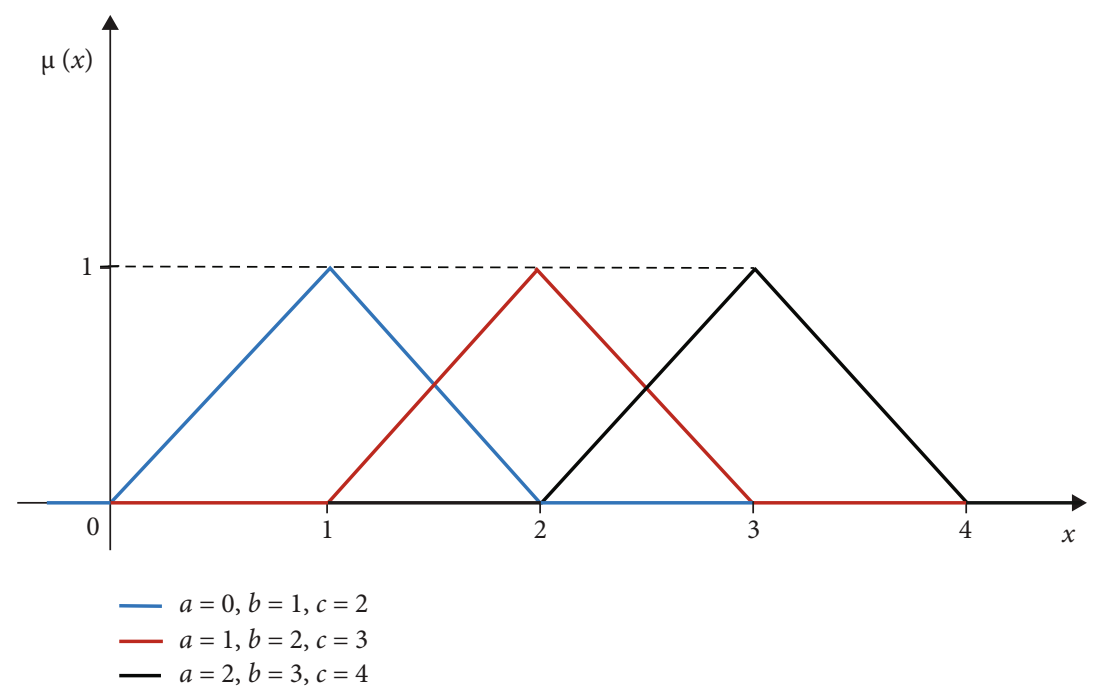

Figure 2: Fuzzy sets with the different triangular membership functions $\mathrm{MF}_{T}$.

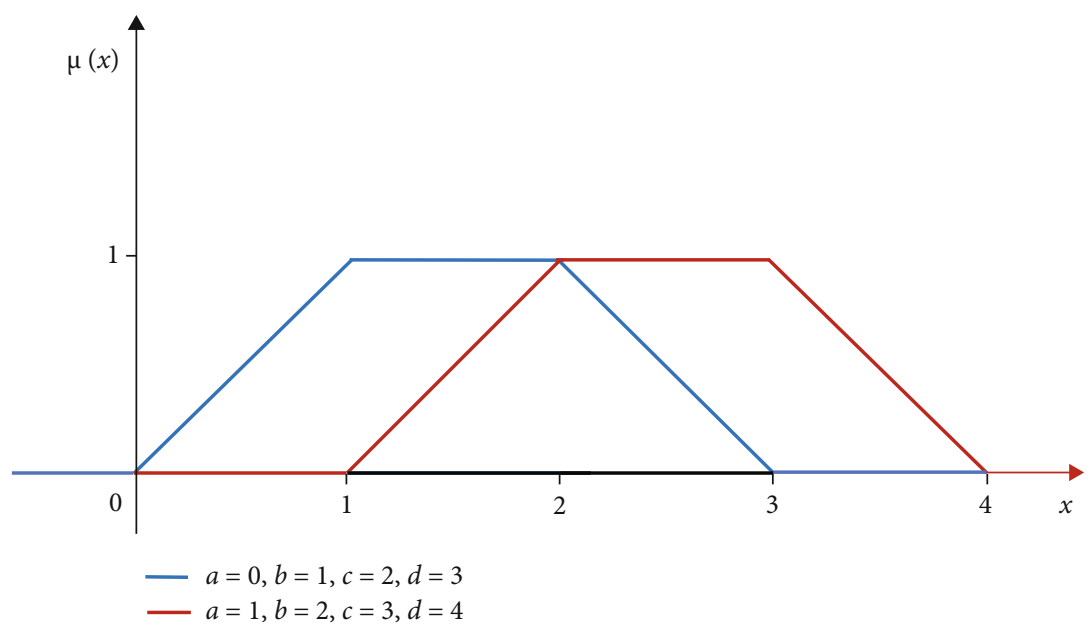

FIGURE 3: Fuzzy sets with different trapezoidal membership functions $\mathrm{MF}_{T p}$.

$$
\tilde{A}=\left\{\left(x, \mu_{\tilde{A}}(x)\right): x \in \mathbb{R} \text { and close to } 0\right\} .
$$

We know that the numbers in the neighborhood of 0 on the real line can be considered close to 0 . Therefore, one may define its membership function as $\mu_{\tilde{A}}$ $=\mathrm{MF}_{T g^{*}}$, considering the center $c=0$ for some $a$ and $b$, depending on the choice of the size for the neighborhood of 0 . For instance, if according to a person numbers like 0.1 and 0.2 are equivalently close to 0 , then one may choose $a=1, b=1$ to represent it via $\mathrm{MF}_{\mathrm{Tg*}}$. On the other hand, if according to another person, 0.4 and 0.45 are also close enough to 0 , then, one may increase the arch to $b=2$, keeping the width as $a=1$ appropriately. In this way, all such fuzzy sets can be defined using $\mathrm{MF}_{T g *}$ by varying the values of $a$ and $b$. The graphical illustration for the fuzzy set $\tilde{A}$ defined using $\mathrm{MF}_{T g *}$ for several values of $a, b$ can be represented by Figure 5 .
Though there are several types of membership functions, the choice highly depends on the distribution of the given data as discussed in the description of generalized bell membership function above. Therefore, the choice of membership function may not be unique [48] and is in fact problem-specific. In terms of practicality, there are some ways in the field of neural networks like genetic algorithms to determine its choice [49].

Remark 3. While fuzzy sets are written as ordered pairs ( $\mathrm{x}, \mu_{\tilde{\mathrm{A}}}(\mathrm{x})$ ) according to Definition 2, the representation for the fuzzy sets can be in various forms depending on the different choices of membership function provided their range lies within 0 and 1 . For special cases such as the triangular membership function (2) and trapezoidal membership function (3), the corresponding fuzzy set $\tilde{A}$ is often denoted by $\langle a, b, c\rangle$ and $\langle a, b, c, d\rangle$, respectively, as standard notations. Here, $b$ and $c$ in the trapezoidal membership function are the values of $x$ where the membership 


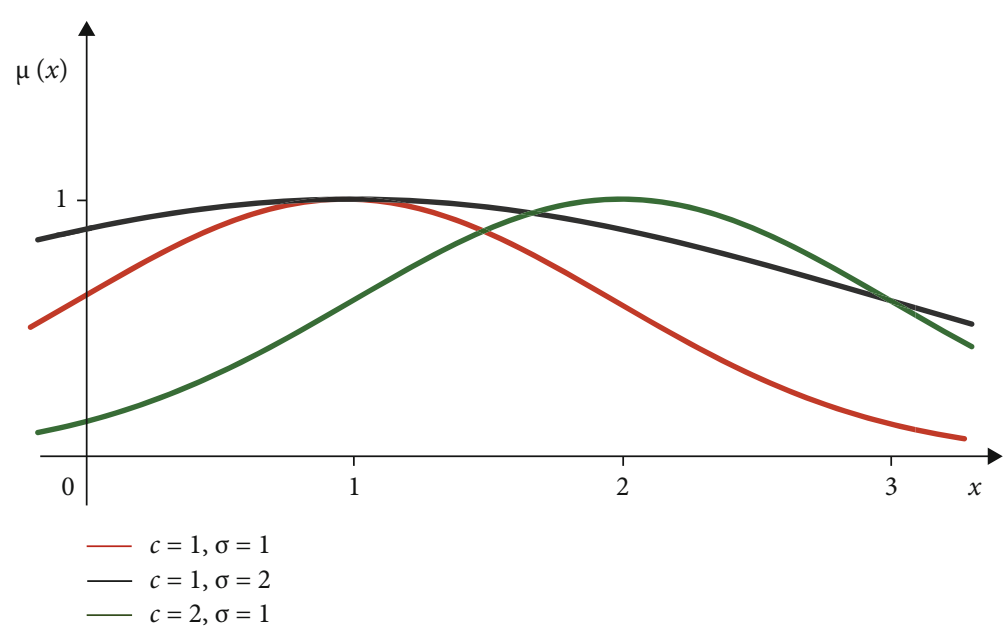

FIGURE 4: Fuzzy sets with different Gaussian membership functions $\mathrm{MF}_{T g}$.

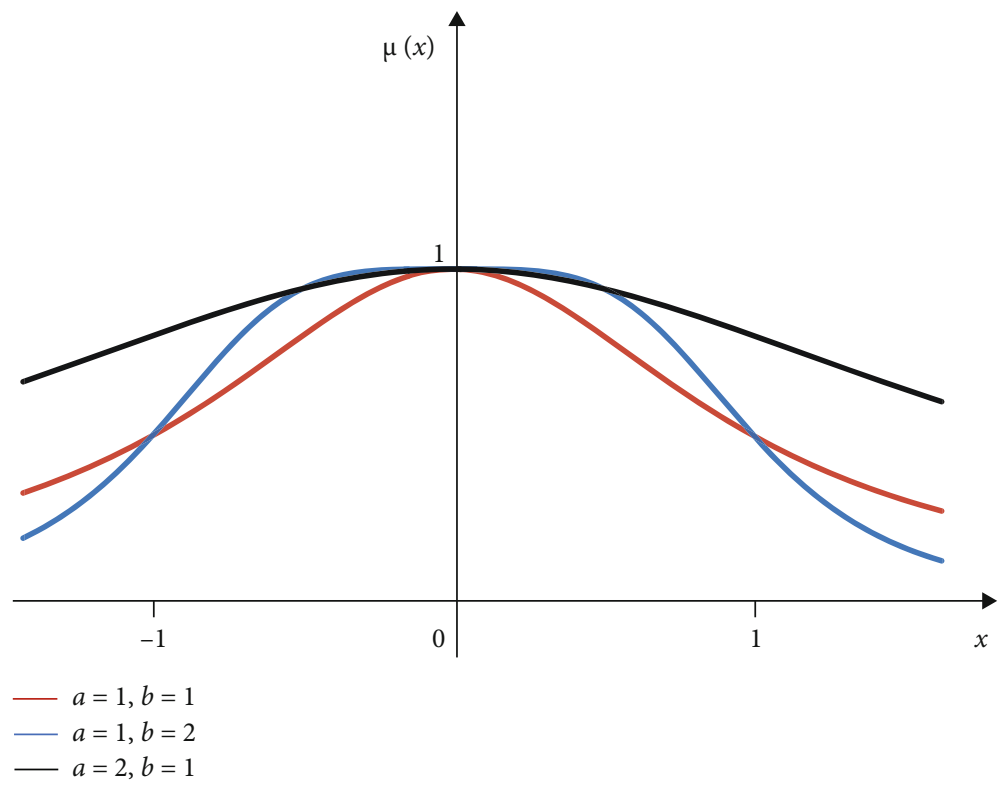

Figure 5: Fuzzy set $\tilde{A}$ with generalized bell membership functions $\mathrm{MF}_{T g *}$ for different values of $a$ and $b$.

function peaks at 1 , and the membership value is 0 at points $a$ and $d$. We shall henceforth denote these fuzzy sets as such.

Although fuzzy sets were introduced in 1965 by Zadeh [50], it was Mizumoto and Tanaka [51] who proved in 1979 that fuzzy sets do not possess addition and multiplication inverses. Subsequently, a collection of fuzzy sets with such membership functions cannot form a field under the ordinary operations of addition and multiplication. This lack of inverses makes it impossible to solve fuzzy equations. In 1980, Yager [52] presented a methodology to obtain approximate solutions based on the highest degree of truth to which the given solution satisfies the given equation. Similarly, numerous studies were conducted to study the properties of membership functions under the crucial notions of convexity and upper semicontinuity so that basic arithmetic operations can be carried out [39, 52-54]. To proceed, we first define fuzzy number and its $\alpha$-level set as follows:

Definition 4 (fuzzy number). Let $X=\mathbb{R}$ be the universe of discourse. We call a fuzzy set $\tilde{A}$ a fuzzy number if its membership function $\mu_{\tilde{A}}: \mathbb{R} \longrightarrow[0,1]$ satisfies the following conditions:

(i) $\mu_{\tilde{A}}$ is normal, i.e., there exists an $x \in \mathbb{R}$ such that $\mu_{\tilde{A}}$ $(x)=1$;

(ii) $\mu_{\tilde{A}}$ is fuzzy convex, i.e.,

$$
\mu_{\tilde{A}}(\lambda x+(1-\lambda) y) \geq \min \left(\mu_{\tilde{A}}(x), \mu_{\tilde{A}}(y)\right),
$$

for all $\lambda \in[0,1]$ and $x, y \in \mathbb{R}$; 


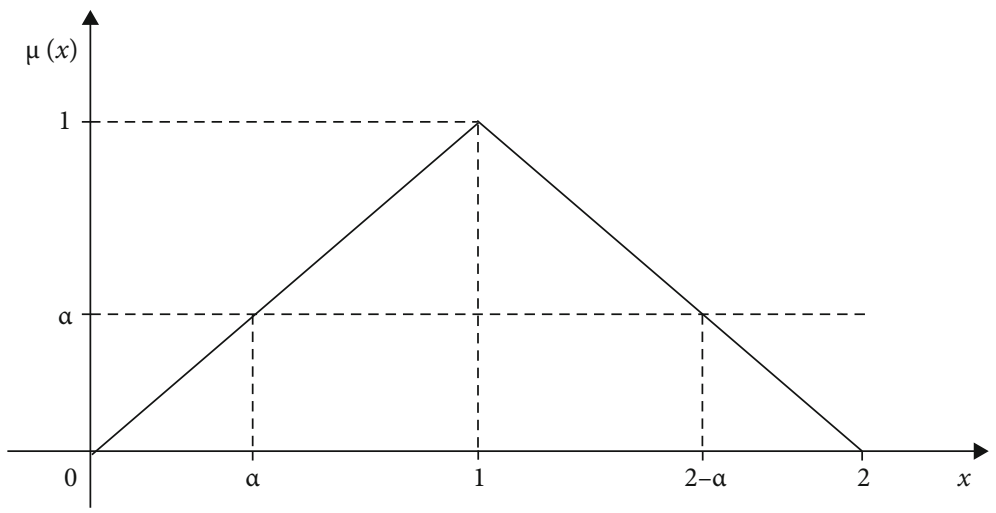

FIGURE 6: Graphical illustration for the $\alpha$-level set of $\tilde{A}$ in Example 2.

(iii) $\mu_{\tilde{A}}$ is upper semicontinuous;

(iv) The closure of the support of $\mu_{\tilde{A}}$ is compact.

In the above definition, upper semicontinuity refers to the concept of upper semicontinous function in a crisp sense stated as follows:

Definition 5 (upper semicontinuity). Let $\mu: \mathbb{R} \longrightarrow[0,1]$ be a real valued function. It is said to be upper semicontinuous at $x^{*} \in \mathbb{R}$ if for each $\varepsilon>0$ there exists $\delta=\delta\left(x^{*}, \varepsilon\right)>0$ such that $\mu(x)<\mu\left(x^{*}\right)+\varepsilon$ whenever $x \in \mathbb{R} \cap N_{\delta}\left(x^{*}\right)$, where $N_{\delta}\left(x^{*}\right)$ denotes delta neighborhood of $x^{*}$. The function $\mu$ is said to be upper semicontinuous on $\mathbb{R}$ if it is upper semicontinuous at every point of $\mathbb{R}$.

We now present the terminology of $\alpha$-level sets that aid in decomposing a fuzzy set into its crisp level sets. In particular, $\alpha$-level sets explicitly express the relation between fuzzy sets and crisp sets [55] that permits us to extend arithmetic operations defined on crisp sets to the case of fuzzy sets, which leads to the reduction in computation cost [54].

Definition 6 ( $\alpha$-level set). Let $\tilde{A}$ be a fuzzy number and $\mu_{\tilde{A}}$ $: \mathbb{R} \longrightarrow[0,1]$ be its membership function. Then, the $\alpha$-level set of $\tilde{\mathrm{A}}$ is defined as

$$
\tilde{A}[\alpha]=\left\{x \in \mathbb{R}: \mu_{\tilde{A}}(x) \geq \alpha\right\} .
$$

for every $\alpha \in[0,1]$.

It can be seen from above that the $\alpha$-level set is a crisp set. We shall henceforth drop tilde from $\tilde{A}[\alpha]$ and denote it by $A[\alpha]$. The $\alpha$-level set $A[\alpha]=\left[A^{l}(\alpha), A^{r}(\alpha)\right]$ is a closed interval in $\mathbb{R}$ for each $\alpha \in[0,1]$, where $A^{l}$ and $A^{r}$ denote the left hand and right hand endpoints of $A[\alpha]$, respectively. Properties of $A^{l}$ and $A^{r}$ (defined for each $\alpha \in[0,1]$ ) are as follows:

(i) $A^{l}:[0,1] \longrightarrow \mathbb{R}$ is a bounded increasing function;

(ii) $A^{r}:[0,1] \longrightarrow \mathbb{R}$ is a bounded decreasing function; (iii) $A^{l}(\alpha) \leq A^{r}(\alpha)$ for every $\alpha \in[0,1]$.

In order to understand the $\alpha$-level set, we now consider the following example.

Example 2. Given a triangular membership function with $a$ $=0, b=1$, and $c=2$ and using the convention in Remark 3 , we write the fuzzy set $\tilde{A}$ as

$$
\tilde{A}=\langle 0,1,2>\text {. }
$$

Note that $\tilde{A}$ satisfies all the properties of a fuzzy number stated in Definition 4, and hence, $\tilde{A}$ is a fuzzy number. We can write the $\alpha$-level set of $\tilde{A}$ as

$$
A[\alpha]=[\alpha, 2-\alpha] \text { for all } \alpha \in[0,1] .
$$

Here, $A^{l}(\alpha)=\alpha$ and $A^{r}(\alpha)=2-\alpha$ are bounded increasing and decreasing functions of $\alpha$, respectively. The graphical illustration for the $\alpha$-level set of $\tilde{A}$ is given in Figure 6 .

Since the $\alpha$-level set $A[\alpha]=\left[A^{l}(\alpha), A^{r}(\alpha)\right]$ represents a closed interval in $\mathbb{R}$ for each $\alpha \in[0,1]$, the operation on $\alpha$ -level sets follows that of interval arithmetic. Pioneered by Moore and Yang [56], interval analysis is one of the major tools in developing algorithms for machine computing dealing with rigorous error bounds. One can refer to $[57,58]$ for operations on interval arithmetic and their properties.

In the following section, we trace out the path of the fuzzy Euler-Lagrange equations, which would turn out to be a fuzzified version of the Euler-Lagrange equation with fuzzy terminologies.

\section{Euler-Lagrange Equation for Variational Problems}

In this section, we first recall the classical approach of variational principle by means of the Euler-Lagrange equation. We scrutinize the strategy and working process of the Euler-Lagrange theorem and compare it with the existing studies concerning fuzzy variational principle to reveal the necessity for its improvement in addressing the current limitations. To begin, we consider the variational problem in the 
following form:

$$
\inf _{u \in X}\left\{I(u)=\int_{\Omega} f(x, u(x), \nabla u(x)) d x\right\}
$$

where $\Omega \subset \mathbb{R}^{n}$ is an open bounded set, $x=\left(x_{1}, x_{2}, \cdots, x_{n}\right) \in$ $\Omega, u: \Omega \longrightarrow \mathbb{R}^{N}$ is a continuously differentiable function, $X=\left\{u \in C^{1}(\Omega): u=u_{0}\right.$ on $\left.\partial \Omega\right\}$, and $f: \Omega \times \mathbb{R}^{N} \times \mathbb{R}^{N \times n}$ $\longrightarrow \mathbb{R}$ is a twice continuously differentiable function. In particular, we seek to minimize the above integral $I(u)$ among all functions $u$ from the admissible space $X$. In the one-dimensional case and under ideal conditions, it is known that we can find the critical points of a function by letting its derivative to be equal to zero. Analogously, this can be done for solutions of smooth variational problems with vanishing functional derivative. To observe it, consider the above (11) in one dimensional case, that is when $n=N=1$ :

$$
\inf _{u \in X}\left\{I(u)=\int_{a}^{b} f\left(x, u(x), u^{\prime}(x)\right) d x\right\}
$$

Assume that $\bar{u}$ is a curve that minimizes the corresponding $I$ in (12). Let $\eta$ be a continuously differentiable function equal to zero on the boundary of $[a, b]$, i.e., $\eta(a$ )$=\eta(b)=0$, such that $\bar{u}+\varepsilon \eta$ takes the same values of $\bar{u}$ on the boundary for every $\varepsilon \in \mathbb{R}$. Set

$$
h(\varepsilon)=I(\bar{u}+\varepsilon \eta)
$$

where $h(\varepsilon)$ has a minimum when $\varepsilon=0$, and hence it must satisfy $h^{\prime}(0)=0$. In other words, the required extremal curve $\bar{u}$ must satisfy

$$
h^{\prime}(0)=\left.\frac{d}{d \varepsilon} I(\bar{u}+\varepsilon \eta)\right|_{\varepsilon=0}=0
$$

Taking expressions (13) and (14) into (12) and differentiating under the integral sign lead to

$$
\int_{a}^{b}\left(\frac{\partial}{\partial u} f\left(x, \bar{u}(x), \bar{u}^{\prime}(x)\right) \eta(x)+\frac{\partial}{\partial u^{\prime}} f\left(x, \bar{u}(x), \bar{u}^{\prime}(x)\right) \eta^{\prime}(x)\right) d x=0 .
$$

Since $f$ is assumed to be a $C^{2}$ function, we integrate by parts to arrive at

$$
\int_{a}^{b}\left(\frac{\partial}{\partial u} f\left(x, \bar{u}(x), \bar{u}^{\prime}(x)\right)-\frac{d}{d x} \frac{\partial}{\partial u^{\prime}} f\left(x, \bar{u}(x), \bar{u}^{\prime}(x)\right)\right) \eta(x) d x=0,
$$

where we have used the convention $\eta(a)=\eta(b)=0$. By the fundamental lemma of calculus of variations, we obtain the Euler-Lagrange equation:

$$
\frac{\partial}{\partial u} f\left(x, \bar{u}(x), \bar{u}^{\prime}(x)\right)-\frac{d}{d x} \frac{\partial}{\partial u^{\prime}} f\left(x, \bar{u}(x), \bar{u}^{\prime}(x)\right)=0 .
$$

By means of the above Euler-Lagrange equation (17) alone, the existence and uniqueness of the solution are not guaranteed. However, if the integrand $f$ is convex, then the existence of minimizer is ensured by Theorem 7 below:

Theorem 7 ([59]). Suppose $f:[a, b] \times \mathbb{R} \times \mathbb{R} \longrightarrow \mathbb{R}$ is $a$ twice continuously differentiable function, $f=f(x, u, \xi)$ and

$$
\inf _{u \in X}\left\{I(u)=\int_{a}^{b} f\left(x, u(x), u^{\prime}(x)\right) d x\right\}=m
$$

where $X=\left\{u \in C^{l}[a, b]: u(a)=\alpha, u(b)=\beta\right\}$.

(i) If (18) has a minimizer $\bar{u} \in X$, then necessarily Equation (17) holds

(ii) Conversely, if $\bar{u}$ satisfies Equation (17) and if $(u, \xi)$ $\longrightarrow f(x, u, \xi)$ is convex for each $x \in[a, b]$, then $\bar{u}$ is a minimizer of (18)

(iii) Furthermore, if the function $(u, \xi) \longrightarrow f(x, u, \xi)$ is strictly convex for every $x \in[a, b]$, then the minimizer of (18), if it exists, is unique

Theorem 7 can also be generalized to higher dimensions. If $u:[a, b] \longrightarrow \mathbb{R}^{N}$, then the Euler-Lagrange equation turns into a system of ordinary differential equations and if $u: \Omega \longrightarrow \mathbb{R}$ where $\Omega$ is an open bounded set in $\mathbb{R}^{n}$, then the Euler-Lagrange equation is a single partial differential equation. If $n, N>1$, then we have a system of partial differential equations.

However, one of the disadvantages of the classical method above is that it was established based on the intuition that every extremal problem will have a solution. Moreover, it is not necessary that the minimizer belongs to $C^{2}[a, b]$ or even $C^{1}[a, b]$, and if $(u, \xi) \longrightarrow f(x, u, \xi)$ is not convex, then the solution of Equation (17) is not essentially an absolute minimizer of $I$ in Equation (18). It may be a local minimizer or a local maximizer (stationary point) of $I$ . Solving the Euler-Lagrange equation in the vectorial case may even lead to complex calculations.

Its limitation on the existence of minimizer is addressed via the direct method that begins by considering a minimizing sequence $\left\{u_{v}\right\} \subset X$ such that $\left\{u_{v}\right\} \rightarrow u$ as $v \longrightarrow \infty$. Under the assumptions of the convexity of $f$ in the last variable and the coercivity condition of $f$

$$
f(x, u, \xi) \geq \beta_{1}|\xi|^{p}+\beta_{2}|u|^{q}+\beta_{3}(x),
$$

for some $\beta_{1}>0, \beta_{2} \in \mathbb{R}, \beta_{3} \in L^{1}(\Omega)$, and $p>q \geq 1$, we prove 
that

$$
I\left(u_{v}\right) \longrightarrow \inf \{I(u)\}=m \text { as } v \longrightarrow \infty
$$

In particular, the functional $I$ in (11) is sequentially weakly lower semicontinuous and hence attains its minimizer.

On the other hand, the functions around us may not always be crisp, hence the need for fuzzy functions to deal with ambiguities and uncertainties. While working well with crisp functions, the variational problems in the calculus of variations cannot be applied to solve ill-posed variational problems with uncertain information. This is because the parameters involved in some functions like time and score are generally uncertain, so their representation using real or crisp numbers leads to an impractical presentation of the real world [60]. To give a practical model, fuzzy numbers can be used as they are adequate for modelling the ambiguity present in the parameters, hence the need for an analytical program to deal with uncertainties that come with fuzzy logic.

In 1987, fuzzy differential equations were first considered by Kaleva [61] to model uncertainties. He gave some existence and uniqueness results for fuzzy differential equations using $\alpha$-level sets. Simultaneously, Seikkala [62] solved a first-order fuzzy initial value problem using Zadeh's principle along with a generalized Lipschitz condition. Using fuzzy numbers, the approach turns out to be effective in solving problems where the initial values are not known precisely, or the nature of errors is not probabilistic due to subjective choices. Similarly, in 1997, Hüllermeier [63] realized that the available knowledge about functional relationships in a dynamical system can be partially available. Therefore, to establish a mathematical modelling for such systems, he utilized vague linguistic information by human experts to design a fuzzy inference system (FIS). The FIS is defined using if-then rules that approximate partially known functions to the desired degree of accuracy. While studying the corresponding initial value problem with fuzzy numbers using the FIS, Hüllermeier observed that the approach gave better results regarding semantical basis and in terms of predictions when compared with other approaches.

Additionally, fuzzy numbers are also studied in reliability science, where subjective judgment and vague data are utilized to ensure that the system performs more effectively. It was observed that the fuzzy logic-based approach overcomes the limitations of traditional reliability approaches such as underestimating the criticality number when failure mode has various effects and neglecting the relative importance of the factors that give the risk priority number more flexibly using linguistic judgments [64, 65].

Fuzzy theory also plays a crucial role in the field of image segmentation. Image segmentation considers the partitions of an image into segments known as pixels to study its characteristics. It is generally used in medical imaging, geographic imaging, and forensic science [1-3]. In 1989, Mumford and Shah [38] formulated the image segmentation problem as an energy functional minimization problem to obtain piecewise-smooth contour $C$ which segments the given gray scale image $f$ using the approximate image $u$. Let $\Omega \subset \mathbb{R}^{2}$ and $f: \Omega \longrightarrow \mathbb{R}$. The proposed energy functional is as follows:

$$
E(u, C)=\int_{\Omega}(u(x)-f(x))^{2} d x+\mu \int_{\Omega \backslash C}|\nabla u|^{2} d x+v|C|,
$$

where $|C|$ denotes the length of the contour $C$, and $\mu$ and $v$ are nonnegative fixed parameters. Later, Chan and Vese [66] considered its simplified model by considering the image $u$ as a piecewise constant function as follows:

$$
E(u, C)=\lambda_{1} \int_{C_{0}}\left|f(x)-c_{1}\right|^{2} d x+\lambda_{2} \int_{C_{I}}\left|f(x)-c_{2}\right|^{2} d x+v|C|,
$$

where $\lambda_{1}, \lambda_{2}>0$ are fixed parameters, $c_{1}$ and $c_{2}$ are constants that define the image intensity of $u$, and $C_{0}$ and $C_{I}$ denote the regions outside and inside the contour $C$, respectively. Following a similar strategy, Krinidis and Chatzis [29] introduced a fuzzy energy-based active contour to deal with the image segmentation of the objects whose boundaries are blur or discontinuous as follows:

$$
\begin{aligned}
E(u, C)= & \lambda_{1} \int_{\Omega}[\mu(x)]^{m}\left|f(x)-c_{1}\right|^{2} d x \\
& +\lambda_{2} \int_{\Omega}[1-\mu(x)]^{m}\left|f(x)-c_{2}\right|^{2} d x+v|C|,
\end{aligned}
$$

where $\mu(x)$ is the degree of membership of $f(x)$ to the inside of the contour $C$ and $m$ is a weighting exponent on each fuzzy membership.

Employing fuzzy theory in the active contouring for image segmentation allows the pixels to belong to multiclusters based on degrees of the membership of each point to the inside or outside the contour. This aids in extracting more information from the original image to enhance the image segmentation abilities $[30,67,68]$. In the above image segmentation problem, the energy functional can be seen as a fuzzy variational problem. Although such problems are often solved numerically by Jacobi iterative techniques or energy difference-based algorithms, the existence of their minimizers is yet to be established. That motivates us to study and advance the theory of fuzzy variational problems.

3.1. Fuzzy Variational Problems. The Euler-Lagrange method discussed at the beginning of this section is relevant only for well-defined crisp functions. It cannot be applied to solve ill-posed problems with uncertain information. The uncertainties that come with fuzzy logic need to be dealt with, requiring the development of an analytical program by considering fuzzy functions instead of crisp functions. Nevertheless, the differences in concepts and definitions do not allow one to substitute the fuzzy function in the EulerLagrange equation in place of the crisp function. For instance, suppose $\tilde{\mu}$ and $\tilde{v}$ are two fuzzy numbers, and we want to know that which number out of these two is larger. It is impossible to answer it using the concept of the crisp 


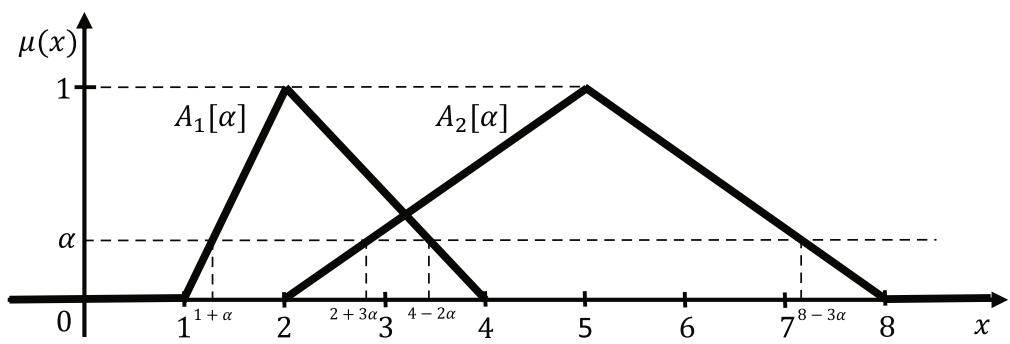

Figure 7: Graphical illustration of the $\alpha$-level sets of $\tilde{A}_{1}$ and $\tilde{A}_{2}$ where $\tilde{A}_{1} \leqslant \tilde{A}_{2}$.

number as we are dealing with fuzzy numbers that do not stand for an individual value but rather a set of possible values. In view of this, we need the concept of partial ordering using $\alpha$-level sets to compare fuzzy numbers, which one can find in Definition 8 below. Similarly, to study fuzzy variational problems, we first define continuity, differentiability, and integrability of a fuzzy function in terms of $\alpha$-level sets $[33,69]$.

Definition 8 (partial order on $\mathscr{F}$ ). Let $\mathscr{F}$ be the set of all fuzzy numbers, and let $\tilde{A}_{1}, \tilde{A}_{2} \in \mathscr{F}$. Then, $\tilde{A}_{1} \leqslant \tilde{A}_{2}$ if $A_{1}^{l}(\alpha) \leq A_{2}^{l}(\alpha)$ and $A_{1}^{r}(\alpha) \leq A_{2}^{r}(\alpha)$ for each $\alpha \in[0,1]$. Similarly, $\tilde{A}_{1} \geqslant \tilde{A}_{2}$ if $A_{1}^{l}(\alpha) \geq A_{2}^{l}(\alpha)$ and $A_{1}^{r}(\alpha) \geq A_{2}^{r}(\alpha)$ for each $\alpha \in[0,1]$. Moreover, $\tilde{A}_{1} \approx \tilde{A}_{2}$ if $\tilde{A}_{1} \leqslant \tilde{A}_{2}$ and $\tilde{A}_{1} \geqslant \tilde{A}_{2}$ hold for each $\alpha \in[0,1]$.

Example 3. Let $A_{1}=\langle 1,2,4\rangle$ and $A_{2}=\langle 2,5,8\rangle$ be two fuzzy numbers and their $\alpha$-level set representations are given in Figure 7. Since $A_{1}^{l}(\alpha) \leq A_{2}^{l}(\alpha)$ and $A_{1}^{r}(\alpha) \leq A_{2}^{r}(\alpha)$ for each $\alpha \in[0,1]$, therefore we conclude that $\tilde{A}_{1} \leqslant \tilde{A}_{2}$.

We would use Definition 6 on $\alpha$-level sets in order to further define the continuity, differentiability, and integrability of a fuzzy-valued function. Therefore, we first recall the definition and the notation for fuzzy-valued function as follows:

Definition 9. Let $\Omega \subseteq \mathbb{R}$. The function $\tilde{f}: \Omega \longrightarrow \mathscr{F}$ is said to be a fuzzy-valued function (or fuzzy function) if for every $x \in \Omega, \tilde{f}(x)$ represents a fuzzy number. as

Using Definition 6 of $\alpha$-level set, $\tilde{f}(x)$ can be represented

$$
f(x)[\alpha]=\left[f^{l}(x, \alpha), f^{r}(x, \alpha)\right]
$$

where

$$
\begin{aligned}
& f^{l}(x, \alpha)=(f(x))^{l}(\alpha)=\min \{f(x)[\alpha]\}, \\
& f^{r}(x, \alpha)=(f(x))^{r}(\alpha)=\max \{f(x)[\alpha]\},
\end{aligned}
$$

are bounded increasing and decreasing functions of $\alpha$, respectively. Here, for each $\alpha \in[0,1]$,

$$
f(x)[\alpha]=\left[f^{l}(x, \alpha), f^{r}(x, \alpha)\right],
$$

represents an $\alpha$-level set.
Definition 10. A fuzzy function $\tilde{f}: \Omega \longrightarrow \mathscr{F}$ is said to be

(i) continuous at $x \in \Omega$ if $f^{l}(x, \alpha)$ and $f^{r}(x, \alpha)$ are continuous functions of $x$ for all $\alpha \in[0,1]$.

(ii) differentiable at $x \in \Omega$ if the derivatives of both $f^{l}$ $(x, \alpha)$ and $f^{r}(x, \alpha)$ with respect to $x$ exist for each fixed $\alpha \in[0,1]$, and the interval $\left[\left(d f^{l} / d x\right)(x, \alpha),(d\right.$ $\left.\left.f^{r} / d x\right)(x, \alpha)\right]$ defines the $\alpha$-level set of a fuzzy number for all $x$ and $\alpha \in[0,1]$. It is denoted by

$$
\frac{d f(x)[\alpha]}{d x}=\left[\frac{d f^{l}}{d x}(x, \alpha), \frac{d f^{r}}{d x}(x, \alpha)\right] .
$$

(iii) integrable with respect to $x \in \Omega$ if both $f^{l}(x, \alpha)$ and $f^{r}(x, \alpha)$ are Riemann integrable functions of $x$, and the interval $\left[\int_{\Omega} f^{l}(x, \alpha), \int_{\Omega} f^{r}(x, \alpha)\right]$ defines the $\alpha$ -level set of a fuzzy number for all $\alpha \in[0,1]$. It is denoted by

$$
\int_{\Omega} f(x)[\alpha] d x=\left[\int_{\Omega} f^{l}(x, \alpha) d x, \int_{\Omega} f^{r}(x, \alpha) d x\right] .
$$

We now consider the following example to observe the above definitions.

Example 4. Let $\tilde{f}:(0,+\infty) \longrightarrow \mathscr{F}$ be a fuzzy function defined by $\tilde{f}(x)=\tilde{C} x^{2}$ where $\tilde{C}$ is a fuzzy number such that $C[\alpha]=[\alpha, 2-\alpha]$ for every $\alpha \in[0,1]$. For each $\alpha \in[0,1]$, we have the $\alpha$-level set $f(x)[\alpha]=\left[\alpha x^{2},(2-\alpha) x^{2}\right]$. Here, $f^{l}(x, \alpha)$ and $f^{r}(x, \alpha)$ are continuous. Moreover, $f^{l}(x, \alpha)$ and $f^{r}(x, \alpha)$ are differentiable; therefore, $f$ is differentiable and

$$
\frac{d f(x)[\alpha]}{d x}=\left[\frac{d f^{l}}{d x}(x, \alpha), \frac{d f^{r}}{d x}(x, \alpha)\right]=[2 \alpha x, 2(2-\alpha) x] .
$$

Now, we let $\tilde{x}=\tilde{x}(t)$ be a fuzzy function of $t \in\left[t_{0}, t_{f}\right] \subseteq \mathbb{R}$ belonging to the set of fuzzy functions whose first derivatives are continuous with respect to $t \in\left[t_{0}, t_{f}\right]$. The fuzzy variational 
problem (FVP) can be expressed as:

$$
\begin{aligned}
& \text { minimize } \quad \tilde{I}(\bar{x}):=\int_{t_{0}}^{t_{f}} \tilde{f}(t, \tilde{x}(t), \tilde{\dot{x}}(t)) d t, \\
& \text { subject to } \tilde{x}\left(t_{0}\right) \approx \tilde{x}_{0}, \tilde{x}\left(t_{f}\right) \approx \tilde{x}_{f} .
\end{aligned}
$$

Here, the integrand $\tilde{f}$ assigns a fuzzy number corresponding to every point $(t, \tilde{x}, \tilde{\dot{x}}) \in \mathbb{R} \times \mathscr{F} \times \mathscr{F}$ where $\tilde{x}$ and $\widetilde{\dot{x}}$ are fuzzy functions of $t \in\left[t_{0}, t_{f}\right]$. Further, we assume that $\tilde{f}$ have continuous first and second partial derivatives with respect to all of its variables. The function $\tilde{x}=\tilde{x}(t)$ is said to be an admissible curve that satisfies the end-point conditions as well as twice continuously differentiable with respect to $t \in\left[t_{0}, t_{f}\right]$. Using all the above concepts and under the assumption that $I^{l}(\tilde{x}, \alpha)$ is taken in the left hand terms (similarly for the right hand terms) such as $x^{l}(t, \alpha)$ and $\dot{x}^{l}(t, \alpha)$, we have the following theorem by Farhadinia [13].

Theorem 11 (fuzzy Euler-Lagrange equations). Let $\tilde{x}_{*}=\tilde{x}_{*}(t)$ be a twice continuously differentiable fuzzy curve satisfying the given endpoints. If $\tilde{x}_{*}$ corresponds to a relative minimum of the fuzzy functional $\tilde{I}$ in (30), then for all $\alpha \in[0,1]$, the following equations necessarily hold:

$$
\frac{\partial f^{l}}{\partial x^{l}}\left(t, \alpha, x_{*}^{l}(t, \alpha), \dot{x}_{*}^{l}(t, \alpha)\right)-\frac{d}{d t}\left(\frac{\partial f^{l}}{\partial \dot{x}^{l}}\left(t, \alpha, x_{*}^{l}(t, \alpha), \dot{x}_{*}^{l}(t, \alpha)\right)\right)=0,
$$

$\frac{\partial f^{r}}{\partial x^{r}}\left(t, \alpha, x_{*}^{r}(t, \alpha), \dot{x}_{*}^{r}(t, \alpha)\right)-\frac{d}{d t}\left(\frac{\partial f^{r}}{\partial \dot{x}^{r}}\left(t, \alpha, x_{*}^{r}(t, \alpha), \dot{x}_{*}^{r}(t, \alpha)\right)\right)=0$.

A direct application of Theorem 11 above can be seen in a fuzzy optimal control model by Roul et al. [4], designed to optimize the production rate so that the total cost is minimized. In contrast to the conventional optimal control models, where all the data is taken in the form of crisp numbers in deterministic imperfect dynamic production models, Roul et al. considered an optimal problem of the minimization of a fuzzy cost functional. The fuzzy variational problem is derived from a dynamic production model with imprecise production due to the variation in raw material quality, workers' efficiencies, machine failure, and imprecise stock level, resulting in the integrand being in terms of fuzzy mappings and the outputs being represented by the corresponding membership functions. When the membership values are set at 1 , the results of the crisp model can also be obtained from the fuzzy model.

In terms of practicality, some of the involved parameters can only be estimated up to an extent due to the presence of uncertainties in real-world optimal control models. Uncertainty and inadequate knowledge are inherent characteristics of modelling mathematical systems. To deal with those uncertainties, real-world problems are often modelled with fuzzy numbers and fuzzy mappings, where the corresponding fuzzy numbers are chosen using prior information, expert knowledge, or genetic algorithm [49]. Such models play an important role in stock trading and map matching algorithm for transport. Chang and Liu [70], for example, considered Takagi-Sugeno-Kang fuzzy-based system using a linear combination of the significant technical index to predict the stock price. While testing on the Taiwan Stock Exchange (TSE) and MediaTek, the proposed method is shown to be an effective trading system for stock price prediction compared to other traditional approaches with an accuracy of 97 $\%$ to $98 \%$ approximately. Similar outcomes were observed in the study of fuzzy map matching algorithms by Quddus et al. [71]. Map matching algorithms are generally based on a probabilistic or topological approach defined using a global positioning system (GPS). While it provides good results, they are not always accurate in high road density regions or in confusing traffic circles. To address this concern, a comprehensive field test was carried out on different road environments using the fuzzy logic-based algorithm. It has been shown that it is more efficient to deal with qualitative terms and linguistic vagueness in comparison to the well-recognized existing map-matching algorithms. The outcome of the study shows that the fuzzy logic-based algorithm determined $99.2 \%$ of the links of the vehicle trajectory correctly.

In mathematical modelling, the concept of differentiability also plays a crucial role in its formulations. It aids in describing the dynamic aspects of the considered system and expresses the functional relationships. In this regard, the concept of fuzzy derivative was first discussed by Pur and Ralescu [72] based on the concept of Hukuhara difference [73]. Since then, Hukuhara differentiability has been widely adopted in studies for proving the existence and uniqueness theorems concerning fuzzy differential equations $[61,62]$. A similar notion of the fuzzy derivative has been studied in metric space (see, for instance, $[72,74-76])$. However, there is a disadvantage of the approach based on the Hukuhara difference, namely it may lead to nondecreasing solutions to the corresponding fuzzy differential equation that can result in increased uncertainties [63]. It was first addressed by Hüllermeier [63] where he used the family of multivalued differential inclusions that considers the fuzzy functions on both sides of the fuzzy differential equations. Although the approach addressed the nondecreasing solution problem, it is worth noting that such derivatives might not provide the result as a fuzzy number. Therefore, to overcome this shortcoming, Buckley and Feuring [77] defined the fuzzy derivative using $\alpha$-level sets and evaluated it using classical differentiation of the endpoints of the $\alpha$-level sets. However, this approach suffers the disadvantage of differential inclusions. In particular, the definition of the derivative is used in the classical sense on $\alpha$-level sets without being defined in the fuzzy sense.

A large number of alternative approaches have been developed over the last few decades to define fuzzy derivatives [78-80]. One of the most recent approaches is the notion of generalized Hukuhara $(\mathrm{gH})$ differentiability [78]. Theorem 11 has also been studied under the $\mathrm{gH}$ differentiability by Fard et al. [12]. Subsequently, it was further extended to derive the fuzzy fractional Euler-Lagrange equations for fuzzy fractional variational problems using the Caputo-type fuzzy fractional and Atangana-Baleanu 
fractional derivatives by Fard and Salehi [42] and Zhang et al. [14], respectively. All such studies employed different differentiability concepts to obtain the necessary optimal conditions for fuzzy variational or fractional variational problems, namely the derivation of the Euler-Lagrange in the fuzzy setting. This motivated Heidari et al. [37] to discuss an approach for the sufficiency conditions regarding the existence of a local minimizer. However, the discussed approach is based on the differential inclusions, studied in the classical sense, by converting the fuzzy variational problem into a crisp variational problem and solving it using the Euler-Lagrange equation. No notions like convexity or strict convexity have been studied in a fuzzy sense without resorting to the Euler-Lagrange equations. Moreover, the uniqueness result has yet been studied. A question that remains to be considered is the existence of those extremals without resorting to the Euler-Lagrange equations since these studies are based on the intuition that every extremal problem will have a solution. Despite that, there has been a lack of extensive literature that analyzes the study of the existence and the uniqueness of minimizers in the fuzzy setting, or more generally, the fuzzy variational principle.

\section{Conclusions and Future Directions}

In the study of fuzzy variational principle, we observed that the necessary optimality conditions had been obtained by following the fundamental pathway established by Euler and Lagrange for problems in the calculus of variations. Considering twice differentiability, the variational principle via the Euler-Lagrange equations provides the necessary optimality conditions under various differentiability notions such as Buckley-Fuering and generalized Hukuhara $(\mathrm{gH})$ derivatives. This was further extended and studied for fuzzy fractional variational principle under Caputo-type and gH Atangana-Baleanu derivatives. Most real-world problems such as image segmentation in medical and geographic imaging and optimal control theory generally focus on solving the corresponding energy integral, which corresponds to a fuzzy variational problem. This reflects the importance of studying and developing the field of fuzzy variational principle for better decision-making. Although decisionmaking is a well-established branch under fuzzy set-theoretic modeling, there is room for development in the fuzzy functional setting which can be applied to complex fuzzy mathematical problems when dealing with functionals. Some of the crucial questions are as follows:

(1) What would the sufficient and necessary conditions be to ensure the existence of minimizers of the fuzzy variational problems?

(2) How would the analogy of the direct method work for fuzzy variational problems?

(3) Are fuzzy Euler-Lagrange equations (31) and (32) applicable to higher dimensions, or do they instead lead to complicated calculations?

Given the questions above, it is crucial to work on the sufficient conditions to ensure the existence and uniqueness of the minimizer. The classical approach of the fuzzy variational problem to find solutions via the Euler-Lagrange equations under twice differentiability seems restrictive. We can consider more relaxed regularity conditions to enlarge the space of admissible functions.

This systematic study also shows that the current approach for seeking a minimizer among functions satisfying the fuzzy Euler-Lagrange equations may lead to partial conclusions and complicated calculations in higher dimensions since the existence of a minimizer is assumed and not established beforehand. This limitation motivates us to study the variational principle to ensure the existence of minimizers for variational problems in the fuzzy setting. A similar analogy of the Euler-Lagrange theorem can be established in the fuzzy setting to obtain sufficient conditions for the existence of minimizer.

In terms of applications to real problems, it is necessary to establish the theory for functionals in fuzzy calculus of variations to find an optimized curve for optimal decisionmaking. For this purpose, one may utilize the calculus of variations' approach to work with higher dimensions without resulting in complex calculations.

\section{Data Availability}

No data were used to support this study.

\section{Conflicts of Interest}

The authors declare that there are no conflicts of interest.

\section{Acknowledgments}

This work was supported by the Malaysian Ministry of Higher Education under Fundamental Research Grant Scheme (FRGS/1/2020/STG06/UPM/02/3 01-01-202296FR) and by Universiti Putra Malaysia under Postgraduate Initiative Grant (GP-IPS/2021/9695100). The authors would like to thank the editor and the reviewers for their helpful comments and suggestions which have led to the improvement of the earlier version of this paper.

\section{References}

[1] D. Cremers, M. Rousson, and R. Deriche, "A review of statistical approaches to level set segmentation: integrating color, texture, motion and shape," International Journal of Computer Vision, vol. 72, no. 2, pp. 195-215, 2007.

[2] D. L. Pham, C. Xu, and J. L. Prince, "Current methods in medical image segmentation," Annual Review of Biomedical Engineering, vol. 2, no. 1, pp. 315-337, 2000.

[3] N. K. Ratha, S. Chen, and A. K. Jain, "Adaptive flow orientation-based feature extraction in fingerprint images," Pattern Recognition, vol. 28, no. 11, pp. 1657-1672, 1995.

[4] J. N. Roul, K. Maity, S. Kar, and M. Maiti, "Optimal control problem for an imperfect production process using fuzzy variational principle," Journal of Intelligent \& Fuzzy Systems, vol. 32, no. 1, pp. 565-577, 2017.

[5] M. Akram, G. Ali, M. A. Butt, and J. C. R. Alcantud, "Novel MCGDM analysis under m-polar fuzzy soft expert sets," 
Neural Computing and Applications, vol. 33, no. 18, pp. 12051-12071, 2021.

[6] G. Ali and M. N. Ansari, "Multiattribute decision-making under Fermatean fuzzy bipolar soft framework," Granular Computing, vol. 6, pp. 1-16, 2021.

[7] G. Ali and M. Sarwar, "Novel technique for group decisionmaking under fuzzy parameterized-rung orthopair fuzzy soft expert framework," Mathematical Problems in Engineering, vol. 2021, Article ID 5449403, 22 pages, 2021.

[8] Z. Ali, T. Mahmood, and G. Santos-García, "Heronian mean operators based on novel complex linear Diophantine uncertain linguistic variables and their applications in multiattribute decision making," Mathematics, vol. 9, no. 21, p. 2730, 2021.

[9] M. Parimala, S. Jafari, M. Riaz, and M. Aslam, "Applying the Dijkstra algorithm to solve a linear Diophantine fuzzy environment," Symmetry, vol. 13, no. 9, p. 1616, 2021.

[10] M. Riaz and M. R. Hashmi, "Linear Diophantine fuzzy set and its applications towards multi-attribute decision-making problems," Journal of Intelligent \& Fuzzy Systems, vol. 37, no. 4, pp. 5417-5439, 2019.

[11] E. Celik, M. Gul, N. Aydin, A. T. Gumus, and A. F. Guneri, “A comprehensive review of multi criteria decision making approaches based on interval type-2 fuzzy sets," KnowledgeBased Systems, vol. 85, pp. 329-341, 2015.

[12] O. S. Fard, A. H. Borzabadi, and M. Heidari, "On fuzzy EulerLagrange equations," Annals of Fuzzy Mathematics and Informatics, vol. 7, no. 3, pp. 447-461, 2014.

[13] B. Farhadinia, "Necessary optimality conditions for fuzzy variational problems," Information Sciences, vol. 181, no. 7, pp. 1348-1357, 2011.

[14] J. Zhang, G. Wang, X. Zhi, and C. Zhou, "Generalized EulerLagrange equations for fuzzy fractional variational problems under gH-Atangana-Baleanu differentiability," Journal of Function Spaces, vol. 2018, Article ID 2740678, 15 pages, 2018.

[15] L. Euler, Methodus inveniendi lineas curvas maximi minimive proprietate gaudentes, Apud Marcum-Michaelem Bousquet, 1744.

[16] M. Giaquinta and S. Hildebrandt, Calculus of Variations I and II, Springer-Verlag, Berlin, 1996.

[17] H. H. Goldstine, A History of the Calculus of Variations from the 17th through the 19th Century, Springer-Verlag, Berlin, 1980.

[18] A. F. Monna, Dirichlet's Principle: A Mathematical Comedy of Errors and Its Influence on the Development of Analysis, Oosthoeck, Utrecht, 1975.

[19] L. Tonelli, "La semicontinuità nel calcolo delle variazioni," Rendiconti del Circolo Matematico di Palermo, vol. 44, no. 1, pp. 167-249, 1920.

[20] E. McShane, "On the necessary condition of Weierstrass in the multiple integral problem of the calculus of variations," Annals of Mathematics, vol. 32, no. 3, pp. 578-590, 1931.

[21] J. Serrin, "On the definition and properties of certain variational integrals," Transactions of the American Mathematical Society, vol. 101, no. 1, pp. 139-167, 1961.

[22] P. Marcellini and C. Sbordone, "Semicontinuity problems in the calculus of variations," Nonlinear Analysis: Theory, Methods \& Applications, vol. 4, no. 2, pp. 241-257, 1980.

[23] I. Fonseca and G. Francfort, "3D-2D asymptotic analysis of an optimal design problem for thin films," Journal für die Reine und Angewandte Mathematik, vol. 1998, no. 505, pp. $173-$ 202, 1998.

[24] P. G. Kirmser and K.-K. Hu, "The shape of the ideal column reconsidered," The Mathematical Intelligencer, vol. 15, no. 3, pp. 62-67, 1993.

[25] A. R. El-Nabulsi, "Quantum field theory from an exponential action functional," Indian Journal of Physics, vol. 87, no. 4, pp. 379-383, 2013.

[26] V. Coverstone-Carroll, J. W. Hartmann, and W. J. Mason, "Optimal multi-objective low-thrust spacecraft trajectories," Computer Methods in Applied Mechanics and Engineering, vol. 186, no. 2-4, pp. 387-402, 2000.

[27] M. Kline, Mathematical Thought from Ancient to Modern Times, vol. 3, Oxford university press, OUP USA, 1990.

[28] E. Kreyszig, "On the calculus of variations and its major influences on the mathematics of the first half of our century. Part I," The American Mathematical Monthly, vol. 101, no. 7, pp. 674-678, 1994.

[29] S. Krinidis and V. Chatzis, "Fuzzy energy-based active contours," IEEE Transactions on Image Processing, vol. 18, no. 12, pp. 2747-2755, 2009.

[30] H. Lv, Z. Wang, S. Fu, C. Zhang, L. Zhai, and X. Liu, “A robust active contour segmentation based on fractional-order differentiation and fuzzy energy," IEEE Access, vol. 5, pp. 77537761, 2017.

[31] L. C. Evans, Partial Differential Equations, American Mathematical Society, 2010.

[32] T. Allahviranloo, "Difference methods for fuzzy partial differential equations," Computational Methods in Applied Mathematics, vol. 2, no. 3, pp. 233-242, 2002.

[33] J. J. Buckley and T. Feuring, "Introduction to fuzzy partial differential equations," Fuzzy Sets and Systems, vol. 105, no. 2, pp. 241-248, 1999.

[34] A. Khastan and R. Rodríguez-López, "An existence and uniqueness result for fuzzy Goursat partial differential equation," Fuzzy Sets and Systems, vol. 375, pp. 141-160, 2019.

[35] H. V. Long, N. T. K. Son, N. T. M. Ha, and L. H. Son, "The existence and uniqueness of fuzzy solutions for hyperbolic partial differential equations," Fuzzy Optimization and Decision Making, vol. 13, no. 4, pp. 435-462, 2014.

[36] N. Mikaeilvand and S. Khakrangin, "Solving fuzzy partial differential equations by fuzzy two-dimensional differential transform method," Neural Computing and Applications, vol. 21, no. S1, pp. 307-312, 2012.

[37] M. Heidari, M. Ramezanzadeh, A. H. Borzabadi, and O. S. Fard, "Solutions to fuzzy variational problems: necessary and sufficient conditions," International Journal of Modelling, Identification and Control, vol. 28, no. 2, pp. 187-198, 2017.

[38] D. B. Mumford and J. Shah, "Optimal approximations by piecewise smooth functions and associated variational problems," Communications on Pure and Applied Mathematics, vol. 42, no. 5, pp. 577-685, 1989.

[39] D. Dubois and H. Prade, "Operations on fuzzy numbers," International Journal of Systems Science, vol. 9, no. 6, pp. 613-626, 1978.

[40] G. Klir and B. Yuan, Fuzzy Sets and Fuzzy Logic, vol. 14, no. 4, 1995, Prentice Hall, New Jersey, 1995.

[41] H.-J. Zimmermann, Fuzzy Set Theory and Its Applications, Springer Science \& Business Media, 2011. 
[42] O. S. Fard and M. Salehi, "A survey on fuzzy fractional variational problems," Journal of Computational and Applied Mathematics, vol. 271, pp. 71-82, 2014.

[43] H. Li and V. C. Yen, Fuzzy Sets and Fuzzy Decision-Making, CRC press, 1995.

[44] L. Lin, X.-H. Yuan, and Z.-Q. Xia, "Multicriteria fuzzy decision-making methods based on intuitionistic fuzzy sets," Journal of Computer and System Sciences, vol. 73, no. 1, pp. 84-88, 2007.

[45] I. Skalna, B. Rebiasz, B. Gawel et al., "Advances in fuzzy decision making," Studies in Fuzziness and Soft Computing, vol. 333, 2015.

[46] D. Driankov, H. Hellendoorn, and M. Reinfrank, An Introduction to Fuzzy Control. Springer Science \& Business, Springer Science \& Business Media, 2013.

[47] A. N. Isizoh, S. O. Okide, A. E. Anazia, and C. D. Ogu, “Temperature control system using fuzzy logic technique," International Journal of Advanced Research in Artificial Intelligence, vol. 1, no. 3, pp. 27-31, 2012.

[48] J. M. Garibaldi and R. I. John, "Choosing membership functions of linguistic terms," in The 12th IEEE International Conference on Fuzzy Systems, vol. 1, pp. 578-583, St. Louis, MO, USA, 2003.

[49] A. Arslan and M. Kaya, "Determination of fuzzy logic membership functions using genetic algorithms," Fuzzy Sets and Systems, vol. 118, no. 2, pp. 297-306, 2001.

[50] L. A. Zadeh, "Fuzzy sets," Information and Control, vol. 8, no. 3, pp. 338-353, 1965.

[51] M. Mizumoto and K. Tanaka, "Some properties of fuzzy numbers," in Advances in Fuzzy Set Theory and Applications, pp. 153-164, North-Holland, 1979.

[52] R. R. Yager, "On the lack of inverses in fuzzy arithmetic," Fuzzy Sets and Systems, vol. 4, no. 1, pp. 73-82, 1980.

[53] J. Dijkman, H. van Haeringen, and S. J. de Lange, "Fuzzy numbers," Journal of Mathematical Analysis and Applications, vol. 92, no. 2, pp. 301-341, 1983.

[54] R. Zhao and R. Govind, "Algebraic characteristics of extended fuzzy numbers," Information Sciences, vol. 54, no. 1-2, pp. 103-130, 1991.

[55] L. A. Zadeh, "The concept of a linguistic variable and its application to approximate reasoning-I," Information Sciences, vol. 8, no. 3, pp. 199-249, 1975.

[56] R. E. Moore and C. Yang, Interval Analysis. Technical Document LMSD-285875, Lockheed Missiles and Space Division, Sunnyvale, CA, USA, 1959.

[57] R. E. Moore, Interval Analysis, Volume 4, Prentice-Hall Englewood Cliffs, 1966.

[58] R. E. Moore, R. B. Kearfott, and M. J. Cloud, Introduction to Interval Analysis, SIAM, 2009.

[59] B. Dacorogna, Introduction to the Calculus of Variations, Imperial College Press, 2009.

[60] M. Verma and K. K. Shukla, "Application of fuzzy optimization to the orienteering problem," Adv. Fuzzy Syst., vol. 2015, article 569248, pp. 1-12, 2015.

[61] O. Kaleva, "Fuzzy differential equations," Fuzzy Sets and Systems, vol. 24, no. 3, pp. 301-317, 1987.

[62] S. Seikkala, "On the fuzzy initial value problem," Fuzzy Sets and Systems, vol. 24, no. 3, pp. 319-330, 1987.

[63] E. Hüllermeier, "An approach to modelling and simulation of uncertain dynamical systems," International Journal of Uncer- tainty, Fuzziness and Knowledge-Based Systems, vol. 5, no. 2, pp. 117-137, 1997.

[64] J. B. Bowles and C. E. Pelaez, "Application of fuzzy logic to reliability engineering," Proceedings of the IEEE, vol. 83, no. 3 , pp. 435-449, 1995.

[65] R. K. Sharma, D. Kumar, and P. Kumar, "Systematic failure mode effect analysis (FMEA) using fuzzy linguistic modelling," International Journal of Quality \& Reliability Management, vol. 22, no. 9, pp. 986-1004, 2005.

[66] T. F. Chan and L. A. Vese, "Active contours without edges," IEEE Transactions on Image Processing, vol. 10, no. 2, pp. 266-277, 2001.

[67] K.-K. Shyu, V.-T. Pham, T.-T. Tran, and P.-L. Lee, "Global and local fuzzy energy-based active contours for image segmentation," Nonlinear Dynamics, vol. 67, no. 2, pp. 1559-1578, 2012.

[68] T.-T. Tran, V.-T. Pham, and K.-K. Shyu, "Image segmentation using fuzzy energy-based active contour with shape prior," Journal of Visual Communication and Image Representation, vol. 25, no. 7, pp. 1732-1745, 2014.

[69] Y.-R. Syau, "On convex and concave fuzzy mappings," Fuzzy Sets and Systems, vol. 103, no. 1, pp. 163-168, 1999.

[70] P.-C. Chang and C.-H. Liu, "A TSK type fuzzy rule based system for stock price prediction," Expert Systems with Applications, vol. 34, no. 1, pp. 135-144, 2008.

[71] M. A. Quddus, R. B. Noland, and W. Y. Ochieng, "A high accuracy fuzzy logic based map matching algorithm for road transport," Journal of Intelligent Transportation Systems, vol. 10, no. 3, pp. 103-115, 2006.

[72] M. L. Puri and D. A. Ralescu, "Differentials of fuzzy functions," Journal of Mathematical Analysis and Applications, vol. 91, no. 2, pp. 552-558, 1983.

[73] M. Hukuhara, "Integration des applications mesurables dont la valeur est un compact convexe," Funkcialaj Ekvacioj, vol. 10, no. 3, pp. 205-223, 1967.

[74] D. Dubois and H. Prade, "On several definitions of the differential of a fuzzy mapping," Fuzzy Sets and Systems, vol. 24, no. 1, pp. 117-120, 1987.

[75] M. Friedman, M. Ma, and A. Kandel, "Fuzzy derivatives and fuzzy Cauchy problems using LP metric," in Fuzzy Logic Foundations and Industrial Applications, pp. 57-72, Springer, 1996.

[76] R. Goetschel Jr. and W. Voxman, "Elementary fuzzy calculus," Fuzzy Sets and Systems, vol. 18, no. 1, pp. 31-43, 1986.

[77] J. J. Buckley and T. Feuring, "Fuzzy differential equations," Fuzzy Sets and Systems, vol. 110, no. 1, pp. 43-54, 2000.

[78] B. Bede and L. Stefanini, "Generalized differentiability of fuzzy-valued functions," Fuzzy Sets and Systems, vol. 230, no. 1, pp. 119-141, 2013.

[79] L. Stefanini, "A generalization of Hukuhara difference and division for interval and fuzzy arithmetic," Fuzzy Sets and Systems, vol. 161, no. 11, pp. 1564-1584, 2010.

[80] L. Stefanini and B. Bede, "Generalized Hukuhara differentiability of interval-valued functions and interval differential equations," Nonlinear Analysis: Theory, Methods \& Applications, vol. 71, no. 3-4, pp. 1311-1328, 2009. 